\title{
Toward epitaxial ternary oxide multilayer device stacks by atomic layer deposition
}

King, Peter J.

2019-03

King , P J , Vehkamäki , M , Mattinen , M , Heikkilä , M J , Mizohata , K, Noh , W , Leskelä , M \& Ritala , M 2019 , ' Toward epitaxial ternary oxide multilayer device stacks by atomic layer deposition ' , Journal of vacuum science \& technology : an official journal of the American Vacuum Society, vol. 37 , no. 2 , 020602 . https://doi.org/10.1116/1.5081997

http://hdl.handle.net/10138/308872

https://doi.org/10.1116/1.5081997

publishedVersion

Downloaded from Helda, University of Helsinki institutional repository.

This is an electronic reprint of the original article.

This reprint may differ from the original in pagination and typographic detail.

Please cite the original version. 


\section{Toward epitaxial ternary oxide multilayer device stacks by atomic layer deposition}

Peter J. King, Marko Vehkamäki, Miika Mattinen, Mikko J. Heikkilä, Kenichiro Mizohata, Wontae Noh, Markku Leskelä, and Mikko Ritala

Citation: Journal of Vacuum Science \& Technology A 37, 020602 (2019); doi: 10.1116/1.5081997

View online: https://doi.org/10.1116/1.5081997

View Table of Contents: https://avs.scitation.org/toc/jva/37/2

Published by the American Vacuum Society

\section{ARTICLES YOU MAY BE INTERESTED IN}

Comparative study on the use of novel heteroleptic cyclopentadienyl-based zirconium precursors with $\mathrm{H}_{2} \mathrm{O}$ and $\mathrm{O}_{3}$ for atomic layer deposition of $\mathrm{ZrO}_{2}$ Journal of Vacuum Science \& Technology A 37, 020912 (2019); https://doi.org/10.1116/1.5079539

Crystalline tungsten sulfide thin films by atomic layer deposition and mild annealing Journal of Vacuum Science \& Technology A 37, 020921 (2019); https://doi.org/10.1116/1.5074153

Review Article: Atomic layer deposition for oxide semiconductor thin film transistors: Advances in research and development

Journal of Vacuum Science \& Technology A 36, 060801 (2018); https://doi.org/10.1116/1.5047237

Atomic layer deposition of cobalt(II) oxide thin films from $\mathrm{Co}(\mathrm{BTSA})_{2}$ (THF) and $\mathrm{H}_{2} \mathrm{O}$

Journal of Vacuum Science \& Technology A 37, 010908 (2019); https://doi.org/10.1116/1.5066638

Surface chemistry of atomic layer deposition: A case study for the trimethylaluminum/water process Journal of Applied Physics 97, 121301 (2005); https://doi.org/10.1063/1.1940727

Conformal deposition of GeTe films with tunable Te composition by atomic layer deposition Journal of Vacuum Science \& Technology A 37, 020907 (2019); https://doi.org/10.1116/1.5079661

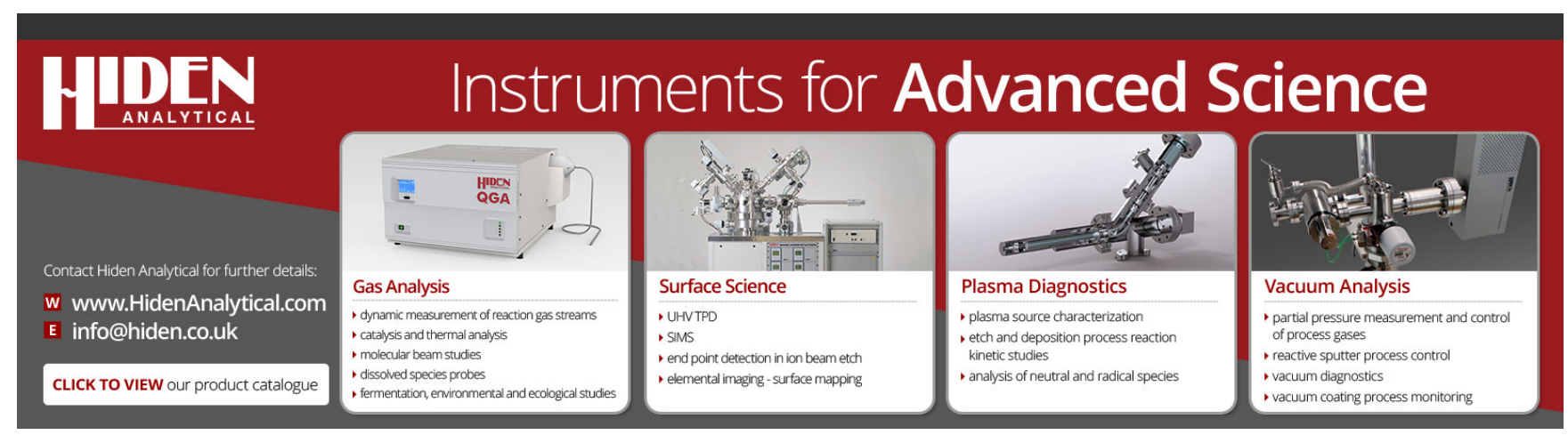




\title{
Toward epitaxial ternary oxide multilayer device stacks by atomic layer deposition
}

\author{
Peter J. King, ${ }^{1}$ Marko Vehkamäki, ${ }^{1}$ Miika Mattinen, ${ }^{1}$ Mikko J. Heikkilä, ${ }^{1}$ \\ Kenichiro Mizohata, ${ }^{2}$ Wontae Noh, ${ }^{3}$ Markku Leskelä, ${ }^{1}$ and Mikko Ritala ${ }^{1, a)}$ \\ ${ }^{1}$ Department of Chemistry, University of Helsinki, P.O. Box 55, FI-00014 Helsinki, Finland \\ ${ }^{2}$ Division of Materials Physics, Department of Physics, University of Helsinki, P.O. Box 43, \\ FI-00014 Helsinki, Finland \\ ${ }^{3}$ Air Liquide Laboratories Korea, Yonsei Engineering Research Park, Suite 176, 50 Yonsei-ro, \\ Seodaemun-gu, Seoul 03722, Republic of Korea
}

(Received 19 November 2018; accepted 7 January 2019; published 28 January 2019)

\begin{abstract}
The authors demonstrate multilayer epitaxial films by atomic layer deposition and postdeposition annealing. Their example features two $\mathrm{ABO}_{3}$ type perovskite oxide films with different materials properties - a conductor $\left(\mathrm{LaNiO}_{3}\right)$ and an insulator $\left(\mathrm{SrTiO}_{3}\right)$ - that can be integrated epitaxially once the geometric interaction between the two oxides' lattices is understood. Once preliminary epitaxial materials had been developed, the pilot multilayer device fabricated was an epitaxial metalinsulator-metal structure with layers of $\sim 5 \mathrm{~nm}$ thickness. This work shows the potential for advanced device types based on epitaxial atomic layer deposited films, assuming that care is taken in the selection of processes and starting substrate. Published by the AVS.

https://doi.org/10.1116/1.5081997
\end{abstract}

\section{INTRODUCTION}

Atomic layer deposition (ALD) has already delivered unparalleled thickness, conformality, and composition control in thin films - enabling the continued scaling of MOSFET and DRAM devices to feature sizes previously thought impossible. ${ }^{1}$ Additionally, ALD is now exploited as a featuredefining tool utilizing the self-aligned layer principle to set the tolerance of device dimension into the nanoscale. ${ }^{2}$ Despite its inventor Suntola originally coining the ALD technique "Atomic Layer Epitaxy,"3 most ALD films are amorphous or polycrystalline. Further device-enabling materials property control is potentially available from films that are singlecrystal and registered to the substrate. The advantages this class of materials offer are improved materials properties and better-defined interfaces for improved device properties. For example, the reduction or elimination of the grain boundary network in ultra-thin high- $\kappa$ layers, and preservation of atomically sharp interfaces, would be expected to reduce leakage current.

Several groups have reported epitaxial ternary and quaternary films by ALD, focusing on materials with exotic device properties like ferromagnetic, ${ }^{4}$ multiferroic, ${ }^{5}$ and superconducting $^{6}$ layers. A group from the University of Texas at Austin has made huge inroads into perovskite integration onto $\mathrm{Si}$ and Ge substrate platforms. ${ }^{7}$ These examples in the literature show that ternary and quaternary epitaxial layers can be produced by ALD, an established manufacturing technique which is already vital for advanced electronic device fabrication.

For the device integration of epitaxial materials, it is necessary to form heterostructures from multilayer stacks of thin films, in order to optimize the materials through the stack

Note: This paper is part of the Special Topic Collection on Complex Oxides.

${ }^{a)}$ Electronic mail: mikko.ritala@ helsinki.fi and across each interface. Such heterostructures have been fabricated by pulsed laser deposition and molecular beam epitaxy in the literature, ${ }^{8,9}$ but metal-insulator-metal (MIM)-type structures are uncommon due to the paucity of options for an epitaxial oxide conductor. In this letter, we describe the use of conductive $\left(\mathrm{LaNiO}_{3}\right)$ and insulating $\left(\mathrm{SrTiO}_{3}\right)$ perovskite oxide thin films to demonstrate the possibility of fabricating epitaxial heterostructures by ALD and annealing steps.

\section{EXPERIMENT}

Depositions were performed at $275^{\circ} \mathrm{C}$ in an ASM F-120 hot-wall flow type reactor with $\mathrm{N}_{2}$ as the process gas and a working pressure of $\sim 10$ mbar. Substrates were $\operatorname{SrTiO}_{3}(100)$ (Pi Kem Ltd.) prepared using a published methodology. ${ }^{10}$ One-side-polished $\mathrm{SrTiO}_{3}(100)$ was heated in air for $1 \mathrm{~h}$ at $1000{ }^{\circ} \mathrm{C}$, cooled, and agitated in de-ionized water for $30 \mathrm{~s}$. This process was performed twice in order to provide atomically flat starting surfaces for deposition. The $\mathrm{LaNiO}_{3}$ process used $\mathrm{La}(2,2,6,6 \text {-tetramethyl-3,5-heptanedionato })_{3}$, $\mathrm{Ni}(2,2,6,6 \text {-tetramethyl-3,5-heptanedionato })_{2}$ (both from Volatec $\mathrm{Oy}$ ), and $\mathrm{O}_{3}$ as precursors. ${ }^{11}$ The $\mathrm{SrTiO}_{3}$ process used $\operatorname{Sr}(1,2,4 \text {-tri-tert-butylcyclopentadienyl) })_{2}$ (Air Liquide), $\mathrm{Ti}(\mathrm{OMe})_{4}$ (Sigma Aldrich), and deionized $\mathrm{H}_{2} \mathrm{O} .{ }^{12}$ The deposition recipes were organized in subcycles of 10 cycles of each binary oxide, e.g., $\left[10\left(\mathrm{Ni}(\text { thd })_{2} / \mathrm{O}_{3}\right)+10\left(\mathrm{La}(\text { thd })_{3} / \mathrm{O}_{3}\right)\right]_{\mathrm{n}}$, with $\mathrm{n}$ supercycles then performed to result in the desired film thickness. This approach has been found to promote stoichiometry in the resulting ternary oxide after the layers intermix during heat treatment. Films were measured using ellipsometry (Film Sense FS-1) and grazing-incidence x-ray diffraction (GIXRD, Rigaku Smartlab). Crystallization temperatures of both oxides were found by variable-temperature XRD in an Anton Paar HTK1200N oven fitted to a PANalytical X'Pert Pro MPD diffractometer. Cross-sectional 
samples for scanning tunneling electron microscope (STEM) analysis were prepared by a Focused Ion Beam instrument. High-angle annular dark field (HAADF)-STEM and energydispersive $\mathrm{x}$-ray spectroscopy (EDS) images for the samples were taken using a probe aberration corrected STEM (FEI Titan Cubed 60-300) operating at $300 \mathrm{keV}$ with a convergence semi-angle of $26.7 \mathrm{mrad}$ for the incident electron probe. Stoichiometry of $\mathrm{LaNiO}_{3}$ was measured by time-of-flight elastic recoil detection analysis (TOF-ERDA) measurements, after which deposition parameters could be optimized. The resistivity was assessed by averaging 5 fourpoint probe measurements made across each film using a Keithley 2400-SourceMeter and Alessi C4S four-point probe head. This average sheet resistance was then converted into resistivity with the film thickness.

\section{RESULTS AND DISCUSSION}

\section{A. Epitaxial $\mathrm{LaNiO}_{3} / \mathrm{SrTiO}_{3}(\mathbf{1 0 0})$}

$\mathrm{LaNiO}_{3}$ is considered a so-called "bad metal"13 - a correlated oxide material that exhibits low resistivity if the oxygen stoichiometry can be preserved during deposition, and the $\mathrm{Ni}$ valence state can be maintained as close to $3+$ as possible. The $\mathrm{LaNiO}_{\mathrm{x}}$ material can display insulating (for majority of nickel being $\mathrm{Ni}^{2+}$ ), semiconducting (for $\mathrm{Ni}^{3+} / \mathrm{Ni}^{2+} \sim 1$ ), and metallic conducting (for majority $\mathrm{Ni}^{3+}$ ) properties. ${ }^{14}$ In the epitaxial MIM structure presented later, $\mathrm{LaNiO}_{3}$ acts as the conductor because epitaxial metals are extremely difficult to produce, especially in stacks where heat treatment is employed to crystallize layers.

$\mathrm{LaNiO}_{3}$ films were amorphous as-deposited and were crystallized by annealing in air for $1 \mathrm{~h}$ at $750{ }^{\circ} \mathrm{C}$. Compositional measurements by TOF-ERDA confirmed the $\mathrm{ABO}_{3}$ stoichiometry of the deposited oxide. Initial attempts to form epitaxial films from layers with a thickness in the order of $30 \mathrm{~nm}$ were unsuccessful as polycrystalline films resulted. It was found that epitaxial film formation only worked for layers below a thickness limit of $\sim 10 \mathrm{~nm}$. Figure 1 is an HAADF-STEM image of such a film with a thickness of $\sim 8.5 \mathrm{~nm}$.

The primitive cell of $\mathrm{LaNiO}_{3}$ has rhombohedral symmetry with the space group $\mathrm{R} \overline{\mathrm{C}} \mathrm{c}$, but the material forms a pseudocubic arrangement on $\mathrm{SrTiO}_{3}(100)$ due to a very close match in the lattice parameters $\left(0.383 \mathrm{~nm}\right.$ for pseudo- $\mathrm{LaNiO}_{3}$, $0.391 \mathrm{~nm}$ for cubic $\mathrm{SrTiO}_{3}$ ). The substrate/film lattice continuity can be seen clearly in Fig. 1 in addition to what appears to be antiphase boundaries; ${ }^{15}$ defects in the lattice that are presumed to relieve the strain of the pseudocubic epitaxial relationship. To make thicker epitaxial $\mathrm{LaNiO}_{3}$ films, a seed layer methodology was necessary where a thin seed layer less than this $\sim 10 \mathrm{~nm}$ thickness limit for epitaxy was deposited and crystallized first, before additional growth was added and crystallized by a second anneal. Using this method with a $5 \mathrm{~nm}$ seed, films of $(5+25) \mathrm{nm}$ were grown and compared with layers that were grown as a $30 \mathrm{~nm}$ continuous deposition. Figure 2(a) is a $\theta / 2 \theta$ XRD measurement for a $30 \mathrm{~nm} \mathrm{LaNiO}_{3}$ film, which can be compared with a

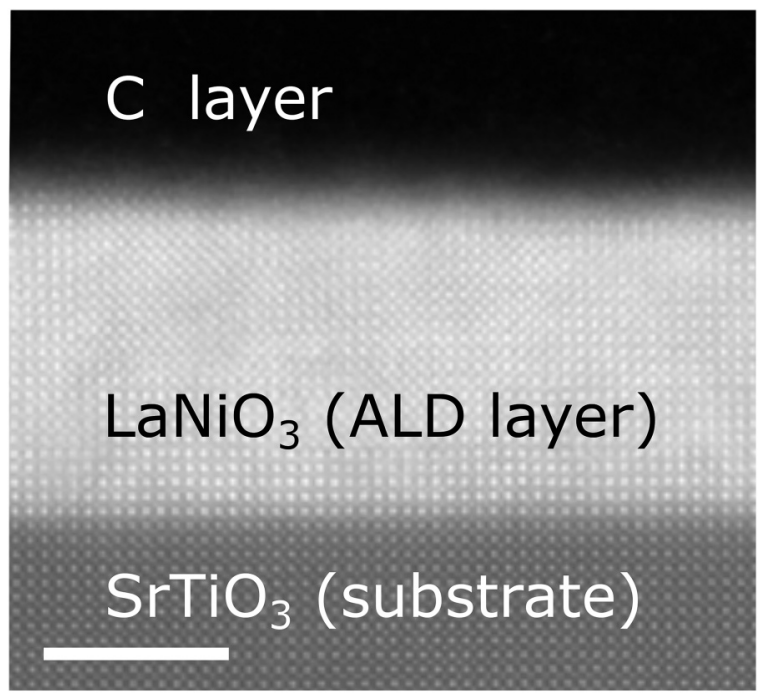

FIG. 1. HAADF-STEM image of an $\sim 8.5 \mathrm{~nm}$ epitaxial $\mathrm{LaNiO}_{3}$ film on $\mathrm{SrTiO}_{3}(100)$ after annealing. The top black area is a carbon film deposited prior to FIB sectioning. Scale bar is $5 \mathrm{~nm}$.

measurement on a seed-layered $(5+25) \mathrm{nm}$ film in Fig. 2(b). The $30 \mathrm{~nm}$ film displays polycrystalline reflections [most obviously the strongest reflection, (110) peak at $\sim 33^{\circ}$ ] while the $(5+25) \mathrm{nm}$ film shows only the pseudocubic $(100)$ and (200) reflections, indicating a highly orientated film product.

To confirm the epitaxial relationship between the substrate and film in the $[5+25] \mathrm{nm}$ sample, a reciprocal space map (RSM) was recorded around the $\mathrm{SrTiO}_{3}(103)$ reflection, as presented in Fig. 3. The $\mathrm{LaNiO}_{3}(103)$ (pseudocubic) reflection is noted in the image, confirming the epitaxial arrangement and demonstrating the close in-plane match between the substrate and film ( $x$ axis in the RSM). The [5+25] nm film had a low resistivity of $\sim 300( \pm 50) \mu \Omega \mathrm{cm}$ which compares well

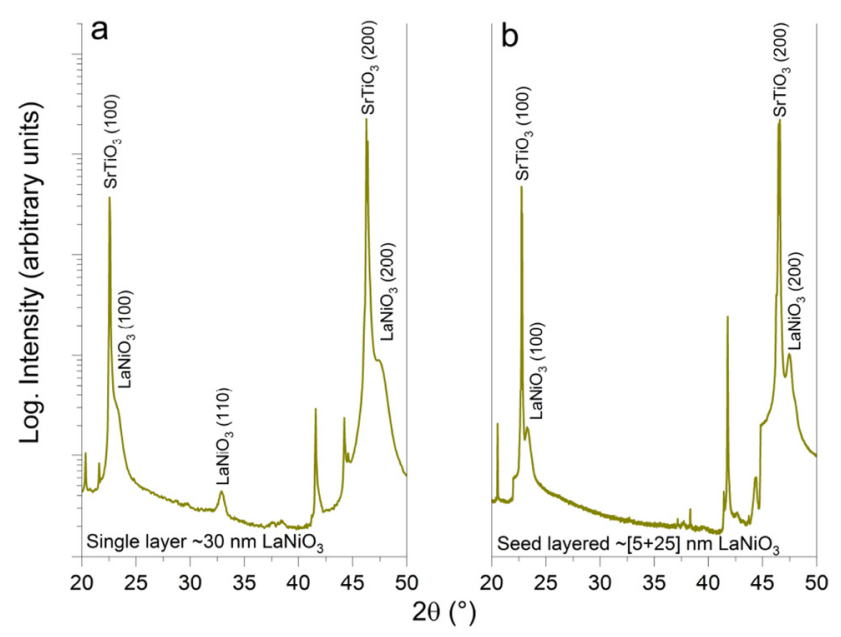

FIG. 2. XRD summary for $\mathrm{LaNiO}_{3}$ films grown on $\mathrm{SrTiO}_{3}(100)$ and annealed. (a) $\theta / 2 \theta$ measurement on a $30 \mathrm{~nm}$ film showing polycrystalline reflections; notably the (110) reflection $\sim 33^{\circ}$. (b) $\theta / 2 \theta$ measurement on a $[5+25] \mathrm{nm}$ film where a $5 \mathrm{~nm}$ seed layer is annealed before subsequent deposition. The sharp, unlabeled peaks in the two diffractograms are from $\mathrm{Cu} \mathrm{K} \beta$ and $\mathrm{W}$ L $\alpha 1$ signals. The data are logarithmic scale in the $y$ axis, $x$ axis units are degrees $2 \theta$. 


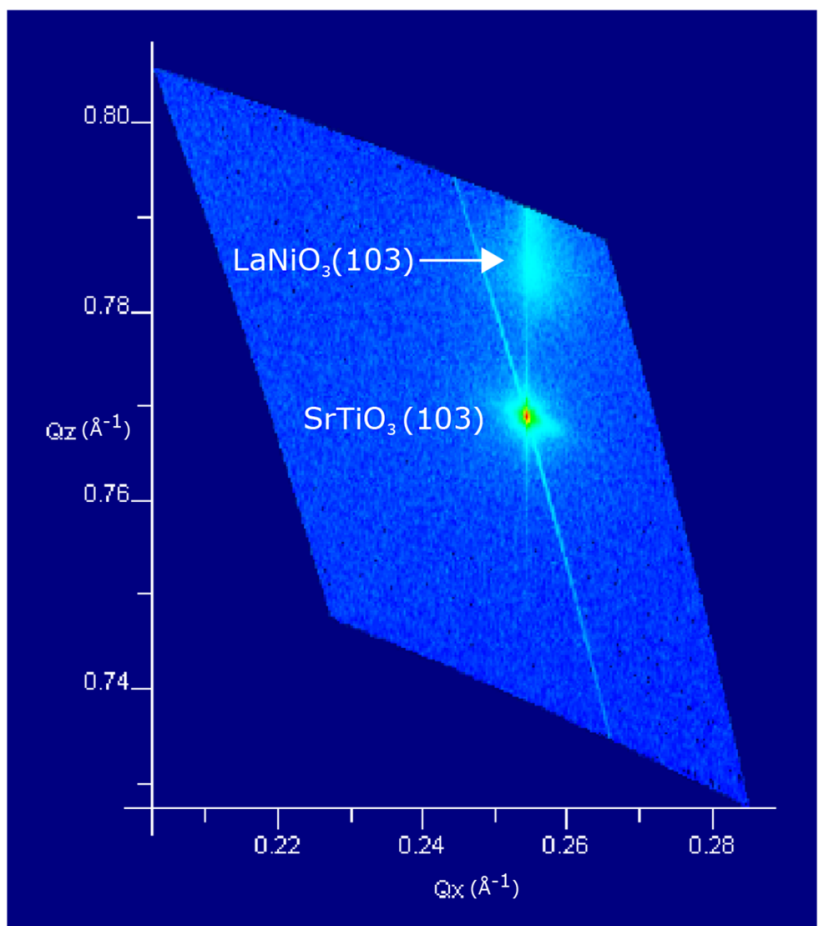

FIG. 3. Reciprocal space map from a seed-layered $[5+25] \mathrm{nm}$ film. The units in both axes are reciprocal Angstroms.

with values for this material in the literature. ${ }^{16,17}$ Thinner films such as those depicted in Sec. III B would be expected to have higher resistivity. The $\sim 8.5 \mathrm{~nm} \mathrm{LaNiO}_{3}$ film shown in Fig. 1 was found to have resistivity closer to $\sim 1500 \mu \Omega \mathrm{cm}$.

\section{B. Multilayer epitaxial $\mathrm{LaNiO}_{3} / \mathrm{SrTiO}_{3} / \mathrm{LaNiO}_{3} / \mathrm{SrTiO}_{3}(\mathbf{1 0 0})$}

Multilayer epitaxial samples were formed by keeping the layer thicknesses $\sim 5 \mathrm{~nm}$ and alternating $\mathrm{LaNiO}_{3}$ and $\mathrm{SrTiO}_{3}$ deposition, with each layer having been annealed at $750{ }^{\circ} \mathrm{C}$ in air after growth to form a crystalline layer. The $\mathrm{SrTiO}_{3}$ layer was found to develop polycrystallinity if the layer thickness was greater than $\sim 10 \mathrm{~nm}$ which is similar to the epitaxy thickness limit seen for the $\mathrm{LaNiO}_{3}$ films grown on $\mathrm{SrTiO}_{3}(100)$ substrates. XRD performed at a range of temperatures showed the crystallization temperature of both of these oxides to be $<600{ }^{\circ} \mathrm{C}$; so the thermal budget for fabrication could potentially be kept lower than that used here. Figure 4 is an HAADF-STEM image of a typical FIB section of the sample, demonstrating that three concurrent epitaxial layers had formed in a heterostructure on the $\mathrm{SrTiO}_{3}$ substrate.

Three FIB sections were analyzed, and common to each was the breakdown of the top $\mathrm{LaNiO}_{3}$ layer in the stack, as can be seen in Fig. 4 (denoted $\mathrm{LaNiO}_{3} / \mathrm{La}_{2} \mathrm{O}_{3}$ in the figure). STEM-EDS measurements showed a $\mathrm{Ni}$ deficiency in this top layer but whether there was an issue with the deposition process itself or this breakdown happened during the annealing step is currently not certain. Careful refabrications of the stack depicted in Fig. 4 have so far all resulted in some polycrystallinity in the topmost layer. Figure 5 presents GIXRD measurements made on a multilayer sample after each layer

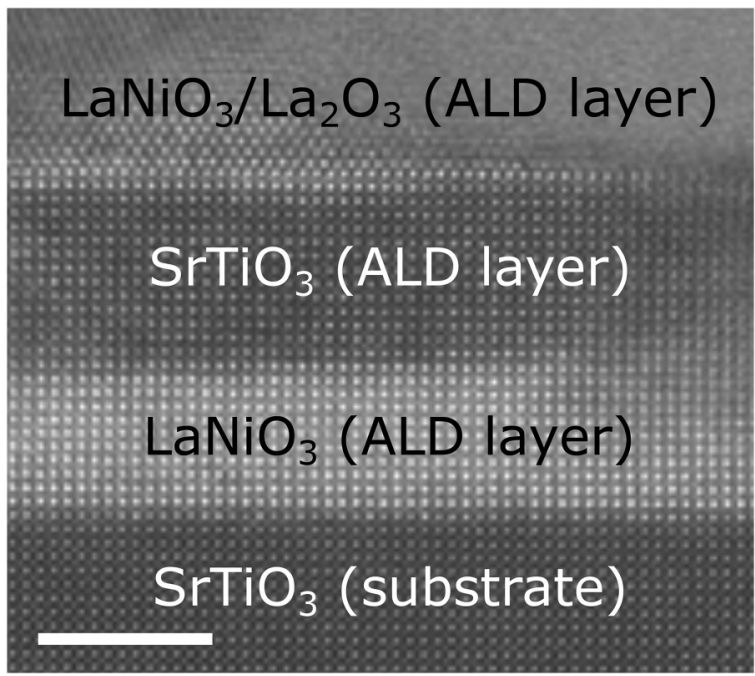

FIG. 4. HAADF-STEM image of epitaxial multilayer sample. $\sim 5 \mathrm{~nm}$ films deposited by ALD and crystallized separately by annealing. Scale bar is $5 \mathrm{~nm}$.

had been deposited and annealed. The GIXRD measurement setup is usually not considered useful for epitaxial films but it was found to be a vital and sensitive tool to check for failure of epitaxy for each individual layer during fabrication. If all layers in the sample crystallize epitaxially, the GIXRD should show only background signal. The small peak at $\sim 30^{\circ}$ in Fig. 5 shows the formation of randomly orientated crystallites after the third layer had been annealed. Scans made over a wider angular range were best fitted to hexagonal $\mathrm{La}_{2} \mathrm{O}_{3}$ XRD pattern. The information in Figs. 4 and 5 is congruent and demonstrates the power of GIXRD to monitor the quality of epitaxial ultra-thin films.

Figure 4 demonstrates that it is possible to produce epitaxial device stacks within a modest thermal budget $\left(275^{\circ} \mathrm{C}\right.$ deposition and $600-750{ }^{\circ} \mathrm{C}$ heat treatment), and it now remains to improve the quality of the samples produced. As the specimens discussed here involved vacuum breaks after each deposition and an air anneal, it is likely that eliminating

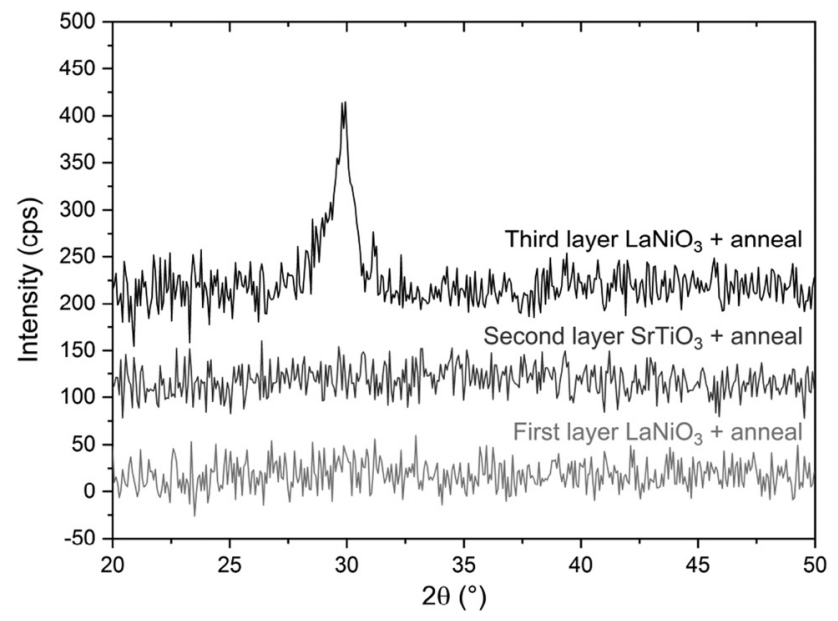

FIG. 5. GIXRD measurements made on an epitaxial MIM sample to check for polycrystallinity after each layer had been annealed. The data are shifted arbitrarily in the $y$ axis, $x$ axis units are degrees $2 \theta$. 
these sources of contamination would improve the interface and film quality. Additionally, it would be interesting to see if such layer stacks could be deposited on a substrate like Ge as has been demonstrated recently by the Texas group in the case of $\mathrm{BaTiO}_{3}{ }^{18}$

\section{SUMMARY AND CONCLUSIONS}

The plausibility of fabricating multilayer epitaxial films by ALD in simple stack device configurations was presented. A pilot MIM-type device was fabricated utilizing ALD processes for $\mathrm{LaNiO}_{3}$ and $\mathrm{SrTiO}_{3}$, combined with postdeposition heat treatment. These results pave the way for the fabrication of advanced ALD-based material devices and are only a preliminary indication of what is likely to be possible with proper fabrication equipment and process knowledge.

\section{ACKNOWLEDGMENTS}

This work was supported by the Finnish Centre of Excellence in Atomic Layer Deposition (Academy of Finland, No. 284623). The authors acknowledge the provision of XRD facilities by Aalto University at OtaNano-Micronova Nanofabrication Centre.

${ }^{1}$ R. W. Johnson, A. Hultqvist, and S. F. Bent, Mater. Today 17, 236 (2014).
${ }^{2}$ J. H.-C. Chen, T. A. Spooner, J. E. Stephens, M. O'Toole, N. LiCausi, B. Kim, S. Narasimha, and C. Child, IEEE IITC (IEEE, Hsinchu, 2017), see https://ieeexplore.ieee.org/document/7968943.

${ }^{3}$ T. Suntola, Mater. Sci. Rep. 4, 261 (1989).

${ }^{4}$ A. R. Akbashev, G. Chen, and J. E. Spanier, Nano Lett. 14, 44 (2014).

${ }^{5}$ C. D. Pham, J. Chang, M. A. Zurbuchen, and J. P. Chang, Chem. Mater. 27, 7282 (2015)

${ }^{6}$ H. H. Sønsteby, T. Aarholt, Ø Prytz, H. Fjellvåga, and O. Nilsen, Chem. Commun. 54, 8253 (2018).

${ }^{7}$ M. D. McDaniel, T. Q. Ngo, S. Hu, A. Posadas, A. A. Demkov, and J. G. Ekerdt, Appl. Phys. Rev. 2, 041301 (2015).

${ }^{8}$ F. Wrobel, A. F. Mark, G. Christiani, W. Sigle, H.-U. Habermeier, P. A. van Aken, G. Logvenov, B. Keimer, and E. Benckiser, Appl. Phys. Lett. 110, 041606 (2017).

${ }^{9}$ Q. Lei et al., npj Quantum Mater. 2, 10 (2017).

${ }^{10}$ J. G. Connell, B. J. Isaac, G. B. Ekanayake, D. R. Strachan, and S. S. A. Seo, Appl. Phys. Lett. 101, 251607 (2012).

${ }^{11}$ H. Seim, H. Mölsä, M. Nieminen, H. Fjellvåg, and L. Niinistö, J. Mater. Chem. 7, 449 (1997).

${ }^{12} \mathrm{M}$. Vehkamäki, "Atomic layer deposition of multicomponent oxide materials," doctoral dissertation(University of Helsinki, 2007), see: http://urn.fi/ URN:ISBN:978-952-10-4452-6.

${ }^{13}$ R. Jaramillo, S. D. Ha, D. M. Silevitch, and S. Ramanathan, Nat. Phys. 10, 304 (2014).

${ }^{14}$ L. Qiao and X. Bi, Europhys. Lett. 93, 57002 (2011).

${ }^{15}$ C. Xu, H. Du, A.J.H.v.d. Torren, J. Aarts, C.-L. Jia, and R. Dittmann, Sci. Rep. 6, 38296 (2016).

${ }^{16}$ A. Li, C. Ge, and P. Lü, Appl. Phys. Lett. 69, 161 (1996).

${ }^{17}$ F. Sánchez, C. Ferrater, C. Guerrero, M. V. García-Cuenca, and M. Varela, Appl. Phys. A 71, 59 (2000).

${ }^{18}$ E. L. Lin, A. B. Posadas, H. W. Wu, D. J. Smith, A. A. Demkov, and J. G. Ekerdt, J. Cryst. Growth 476, 6 (2017). 